\title{
Comparative proteomic analysis of pepper (Capsicum annuum L.) seedlings under selenium stress
}

\author{
Chenghao Zhang ${ }^{1,2}$, Baoyu Xu ${ }^{1}$, Wei Geng ${ }^{3}$, Yunde Shen ${ }^{4}$, Dongji Xuan ${ }^{4}$, Qixian Lai ${ }^{5}$, Chenjia Shen ${ }^{6}$, Chengwu \\ Jin ${ }^{\text {Corresp., } 7}$, Chenliang Yu ${ }^{\text {Corresp. } 8}$ \\ 1 Institute of Agricultural Equipment, Zhejiang Academy of Agricultural Sciences, Hangzhou, Zhejiang, China \\ ${ }^{2}$ Key Labortatory of Creative Agricultrue, Ministry of Agriculture,, Zhejiang Academy of Agricultural Science, Hangzhou, Zhejiang, China \\ 3 Vegetable Research Institute, Zhejiang Academy of Agricultural Sciences, Hangzhou, Zhejiang, China \\ 4 College of Mechanical and Electrical Engineering, Wenzhou University, Wenzhou, Zhejiang, China \\ ${ }^{5}$ Key Labortatory of Creative Agricultrue, Ministry of Agriculture,, Zhejiang Academy of Agricultural Sciences, Hangzhou, Zhejiang, China \\ 6 College of Life and Environmental Science, Hangzhou Normal University,, Hangzhou, Zhejiang, China \\ 7 School of Food Engineering, Ludong University, Yantai, Shandong, China \\ 8 Institute of Agricultural Equipment, Zhejiang Academy of Agricultural Science, Hangzhou, Zhejiang, China \\ Corresponding Authors: Chengwu Jin, Chenliang Yu \\ Email address: jinchwu@Idu.edu.cn, 21007030@zju.edu.cn
}

Selenium (Se) is an essential trace element for human and animal health. Se fertilizer has been used to increase the Se content in crops to meet the Se requirements in humans and animals. To address the challenge of Se poisoning in plants, the mechanisms underlying Se-induced stress in plants must be understood. Here, to elucidate the effects of Se stress on the protein levels in pepper, we used an integrated approach involving tandem mass tag labeling, HPLC fractionation, and mass spectrometry-based analysis. A total of 4,693 proteins were identified, 3,938 of which yielded quantitative information. Among them, the expression of 172 proteins was up-regulated, and the expression of 28 proteins was downregulated in the Se/mock treatment comparison. According to the above data, we performed a systematic bioinformatics analysis of all identified proteins and differentially expressed proteins (DEPs). The DEPs were most strongly associated with the terms 'metabolic process', 'posttranslational modification, protein turnover, chaperones,' and 'protein processing in endoplasmic reticulum' according to GO, KOG classification, and KEGG enrichment analysis, respectively. Furthermore, several heat shock proteins were identified as DEPs. These results provide insights that may facilitate further studies on the pepper proteome expressed downstream of the Se stress response. Our data revealed that the responses of pepper to Se stress involve various pathways. 


\section{Comparative Proteomic Analysis of Pepper (Capsicum 2 annuum L.) Seedlings under Selenium Stress}

3

4 Chenghao Zhang ${ }^{1,2}$, Baoyu Xu${ }^{1}$, Wei Geng ${ }^{3}$, Yunde Shen ${ }^{4}$, Dongji Xuan ${ }^{4}$,Qixian Lai ${ }^{2}$, Chenjia 5 Shen $^{5}$, Chengwu Jin ${ }^{6 *}$ and Chenliang $\mathrm{Yu}^{1 *}$

61 Institute of Agricultural Equipment, Zhejiang Academy of Agricultural Sciences, Hangzhou, $7 \quad$ Zhejiang, China;

82 Key Labortatory of Creative Agricultrue, Ministry of Agriculture, Zhejiang Academy of

9 Agricultural Sciences, Hangzhou, Zhejiang, China;

103 Vegetable Research Institute, Zhejiang Academy of Agricultural Sciences, Hangzhou, 11 Zhejiang, China;

124 College of Mechanical and Electrical Engineering, Wenzhou University, Wenzhou, 13 Zhejiang, China;

145 College of Life and Environmental Science, Hangzhou Normal University, Hangzhou , 15 Zhejiang, China;

166 School of Food Engineering, Ludong University, Yantai, Shandong, China;

17 Corresponding Author:

18 Chengwu Jin

19186 Hongqizhong Road, Shandong Province, 264025, China

20 Email address: jinchwu@hotmail.com

21 Chenliang Yu

22198 Shiqiao Road, Zhejiang Province,310021, China

23 Email address:21007030

24

25

26

27

28

29

30

31

32

\section{Abstract}

Selenium (Se) is an essential trace element for human and animal health. Se fertilizer has been used to increase the Se content in crops to meet the Se requirements in humans and animals. To address the challenge of Se poisoning in plants, the mechanisms underlying Se-induced stress in plants must be understood. Here, to elucidate the effects of Se stress on the protein levels in pepper, we used an integrated approach involving tandem mass tag labeling, HPLC fractionation, and mass spectrometry-based analysis. A total of 4,693 proteins were identified, 3,938 of which yielded quantitative information. Among them, the expression of 172 proteins was up-regulated, and the expression of 28 proteins was down-regulated in the Se/mock treatment comparison. 
33

34

35

36

37

38

39

40

41

42

43

44

45

46

47

48

49

50

51

52

53

54

55

56

57

58

59

60

61

62

63

64

65

66

67

68

69

70

71

According to the above data, we performed a systematic bioinformatics analysis of all identified proteins and differentially expressed proteins (DEPs). The DEPs were most strongly associated with the terms 'metabolic process', 'posttranslational modification, protein turnover, chaperones,' and 'protein processing in endoplasmic reticulum' according to GO, KOG classification, and KEGG enrichment analysis, respectively. Furthermore, several heat shock proteins were identified as DEPs. These results provide insights that may facilitate further studies on the pepper proteome expressed downstream of the Se stress response. Our data revealed that the responses of pepper to Se stress involve various pathways.

\section{Introduction}

Selenium (Se) is a trace element that is essential for human and animal health, and it is an active component of numerous enzymes in human metabolism (Sager, 2006; Semnani et al., 2010). Because of its important protective effects in animals and plants, many studies on Se have been conducted in a broad range of fields including medicine, agriculture, and nutrition (Chen et al., 2002; Thavarajah, Ruszkowski \& Vandenberg, 2008). Although there is no direct evidence that Se is necessary for plant growth, Se plays a key role in plant growth and development. Se can improve antioxidant enzyme activity and enhance the tolerance of Rumex patientia $x R$. tianshanicus seedlings to salt stress (Kong, Wang \& Bi, 2005). In addition, Se enhances plant resistance to abiotic stresses, including heavy metals (Kumar et al., 2012), waterlogging (Wang, 2011), chilling (Chu, Yao \& Zhang, 2010), high temperature (Djanaguiraman, Prasad \& Seppanen, 2010) and drying (Pukacka, Ratajczak \& Kalemba, 2011). Se also plays a critical role in plant resistance to biological stress. Plants with high Se content in grasslands can resist invasion by herbivores (Quinn et al., 2008).

The properties of Se facilitate the formation of stable compound structures with multiple oxidation states $(+2,+4$, and +6$)$, covalent bonding to non-metals (such as carbon), and strong coordination with metals such as cadmium (Fernandes et al., 2018). The main forms of Se taken up by plants are selenate (VI) and selenite (IV), whereas the Se forms in soil are influenced by the soil $\mathrm{pH}$ and oxidation potential (Elrashidi et al., 1987). The uptake and transport mechanisms of the two major valences of $\mathrm{Se}\left(\mathrm{SeO}_{4}{ }^{2-}\right.$ and $\left.\mathrm{SeO}_{3}{ }^{2-}\right)$ in soil differ (White, 2016). The chemical properties of selenate and sulfate are similar (Shibagaki et al., 2002). These compounds are antagonistic during plant uptake, and the sulfate transporter regulates the uptake of selenite (Shibagaki et al., 2002; El Kassis et al., 2007; White, 2016). Currently, the mechanism of selenite absorption by plants is unclear. Most studies suggest that the mechanism of absorption of selenite is similar to that of phosphate, but selenite absorption is negatively correlated with phosphate absorption (Zhang et al., 2014; Song et al., 2017).

At present, Se poisoning incidents in plants have rarely been reported. Preliminary studies have shown that the toxic effect of Se on plants is similar to that of heavy metals to some extent and can hinder plant growth and metabolism. In agricultural environments, excessive Se has been found to decrease radish seeds by $14 \%$ and radish yield by $8-9 \%$ ( Hladun et al., 2013). Se 
72 stress in barley hinders plant growth and significantly decreases fresh weight, water content, and

73 photosynthetic capacity (Molnárová \& Fargašová, 2009). Paciolla et al. (2011) have observed

74 that Se treatment (8-16 mg/L) significantly inhibits barley germination (Paciolla, De Leonardis

75 \& Dipierro, 2011). In addition, Se (4-6 mg/L) significantly inhibits root and bud growth in

76 soybean seedlings, whereas root growth in lettuce and ryegrass is completely inhibited even at a

77 concentration of $1 \mathrm{mg} / \mathrm{L}$ (Hartikainen et al., 1997; Aggarwal et al., 2011). The above results

78 demonstrate that Se causes toxicity in plants. However, current understating of the molecular

79 mechanism of Se toxicity remains limited (El-Ramady et al., 2015; Galinha et al., 2015; Jia et

80 al.,2019). Elemental Se and Se compounds are increasingly accumulating in surface soil and

81 water. Excessive amounts of Se pose a potential risk in agricultural production (Kuppusamy et al.,

82 2017; Jia et al., 2019).

83

84

85

86

87

88

89

90

91

92

93

94

95

96

97

98

99

100

101

102

103

104

105

106

107

108

109

110

Pepper (Capsicum annuum L.), an economically important vegetable in the Solanaceae family, has been used as a spice in China and Korea for decades (Choi et al,2005). Recently, a novel tandem mass spectrometry (MS/MS)-based tandem mass tag (TMT) labeling strategy was developed for large-scale protein quantification (Hao et al,2017; Xu et al,2017). Relatively limited proteomic data on pepper under Se stress have been reported. In the present study, we used a TMT labeling-based quantitative proteomics approach to identify differentially expressed proteins (DEPs) under Se treatment. Our data enabled the identification and exploration of the roles of candidate proteins associated with Se stress resistance.

\section{Materials \& Methods}

\section{Plant Materials and Se Treatments}

Pepper seeds (C. annuum 8 \#, a cultivar provided by pepper breeding group in Fujian Agriculture and Forestry University) were sterilized with $1 \%$ sodium hypochlorite for $30 \mathrm{~min}$ and grown in steam-sterilized soil. The seedlings were grown in a greenhouse under the following conditions: $12 \mathrm{~h}$ light $(150 \mu \mathrm{m} 2 \mathrm{~s}-1)$ at $26^{\circ} \mathrm{C}, 12 \mathrm{~h}$ dark at $23^{\circ} \mathrm{C}$, and relative humidity of $60 \%$. Seedlings were irrigated with half-strength Hoagland solution (pH 5.6). Pepper plants at the four true leaf stages were used for Se treatment. Seedlings were sprayed with half-strength Hoagland solution containing 0 or $100 \mathrm{ppm} \mathrm{Na}_{2} \mathrm{SeO}_{4}$. After $24 \mathrm{~h}$, the shoots were collected for protein extraction.

\section{Protein Extraction and Trypsin Digestion}

Samples were removed from storage at $-80^{\circ} \mathrm{C}$, and fixed amounts of tissue samples were ground to powder while liquid nitrogen was added. The samples in each group were treated with four volumes of phenol extraction buffer (containing $10 \mathrm{mM}$ dithiothreitol, $1 \%$ protease inhibitor, and $2 \mathrm{mM}$ EDTA), then sonicated three times on ice with a high intensity ultrasonic processor (Scientz, Ningbo, China). The supernatant was centrifuged for $10 \mathrm{~min}$ at $4^{\circ} \mathrm{C}$ and 5,500 $\mathrm{g}$ with an equal volume of Tris equilibrium phenol. The supernatant was collected and precipitated overnight with five volumes of $0.1 \mathrm{M}$ ammonium acetate/methanol. The protein precipitate was washed with methanol and acetone successively. Finally, the precipitate was re-dissolved in $8 \mathrm{M}$ 
111 urea, and the protein concentration was determined with a BCA kit (code P0010, Beyotime,

112

113

114

115

116

117

118

119

120

121

122

123

124

125

126

127

128

129

130

131

132

133

134

135

136

137

138

139

140

141

142

143

144

145

146

147

148

149

150

Beijing, China) according to the manufacturer's instructions.

For digestion, the final concentration of dithiothreitol in protein solution was $5 \mathrm{mM}$, and reduction was performed by incubation at $56^{\circ} \mathrm{C}$ for 30 minutes. The mixture was then alkylated with $11 \mathrm{mM}$ iodoacetamide at room temperature for 15 minutes. Finally, the urea concentration of the sample was diluted to less than $2 \mathrm{M}$ by addition of $100 \mathrm{mM}$ triethyl ammonium bicarbonate. Trypsin was added at a mass ratio of 1:50 (trypsin:protein), and enzymatic hydrolysis was carried out overnight at $37^{\circ} \mathrm{C}$. Trypsin was then added at a mass ratio of 1:100 (trypsin:protein), and enzymatic hydrolysis continued for $4 \mathrm{~h}$.

\section{TMT Labeling and HPLC Fractionation}

The trypsinized peptide segments were desalted with a Strata X C18 column (Phenomenex, Torrance, US) and then freeze-dried under vacuum. The peptides were dissolved in $0.5 \mathrm{M}$ triethyl ammonium bicarbonate and labeled with a TMT kit (ThermoFisher, Shanghai, China) according to the manufacturer's instructions. The procedure was as follows: the labeled reagent was dissolved in acetonitrile after thawing, incubated at room temperature for $2 \mathrm{~h}$ after mixing with the peptide segments, then desalinated after mixing with the labeled peptide segment and freeze-dried in a vacuum.

Peptide segments were classified with high $\mathrm{pH}$ reverse-phase high performance liquid chromatography with an Agilent 300 Extend C18 column (5 $\mu \mathrm{m}$ diameter, $4.6 \mathrm{~mm}$ inner diameter, $250 \mathrm{~mm}$ length) (Agilent, Shanghai, China). The gradient of peptide segments was 8$32 \%$ acetonitrile ( $\mathrm{pH} 9.0$ ), and more than 60 minutes was required to separate the peptide segments into 60 components. The peptide segments were then merged into 18 components, which were then vacuum freeze-dried.

\section{LC-MS/MS Analysis}

The tryptic peptides were dissolved in $0.1 \%$ formic acid (solvent A) and directly loaded onto an Acclaim PepMap 100 reverse-phase pre-column (ThermoFisher, Shanghai, China). The gradient comprised an increase from $6 \%$ to $23 \%$ solvent B (0.1\% formic acid in $98 \%$ acetonitrile) over 26 min, from $23 \%$ to $35 \%$ in $8 \mathrm{~min}$, and to $80 \%$ in $3 \mathrm{~min}$; then the concentration was held at $80 \%$ for the last $3 \mathrm{~min}$. All steps were performed at a constant flow rate of $500 \mathrm{~nL} / \mathrm{min}$ on an EASYnLC 1000 ultra-high performance liquid chromatography system.

After equilibration, the peptides were ionized with an NSI ion source and analyzed by MS/MS (Q ExactiveTM mass spectrometer; ThermoFisher, Shanghai, China) coupled online to ultra-high performance liquid chromatography. The ion source voltage was set to $2.0 \mathrm{kV}$, and the parent ions of peptide segments and their secondary fragments were detected and analyzed with a high resolution Orbitrap. The scanning range of the primary MS was set to $350-1,800 \mathrm{~m} / \mathrm{z}$, and the scanning resolution was set to 70,000 . The scanning range of the secondary MS was set to $100 \mathrm{~m} / \mathrm{z}$, and the scanning resolution of the secondary MS was set to 17,500 . The data acquisition mode used a data-dependent scanning program; that is, the first 20 peptide ions with 
151 the highest signal intensity were selected to enter the higher collision dissociation collision pool

152

153

154

155

156

157

158

159

160

161

162

163

164

165

166

167

168

169

170

171

172

173

174

175

176

177

178

179

180

181

182

183

184

185

186

187

188

189

in turn after the first scan, and the fragmentation energy was $28 \%$ for fragmentation. Similarly, secondary MS analysis was carried out sequentially. To improve the efficiency of MS, AGC was set to $5 \mathrm{E} 4$, the signal threshold was set to $2 \mathrm{E} 4$, the maximum injection time was set to $100 \mathrm{~ms}$, and the dynamic elimination time of MS/MS scanning was set to $30 \mathrm{~s}$ to avoid repeated scanning of parent ions.

\section{Database Search}

The resulting MS/MS data were processed and searched against a public proteome Capsicum annuum database (https://www.uniprot.org/uniprot/?query=proteome:UP000222542) with the Maxquant search engine (v.1.5.2.8, https://maxquant.org/) concatenated with a reverse decoy database. The retrieval parameter settings were as follows: trypsin/ $\mathrm{P}$ was used for digestion; the number of missing digestion sites was set to two; and the minimum length of peptide segments was set to seven amino acids. The maximum number of modifications of the peptide segment was set to five. The mass error tolerance of the primary parent ions of the first search and main search was set to $20 \mathrm{ppm}$ and $5 \mathrm{ppm}$, respectively. The mass error tolerance was $0.02 \mathrm{Da}$.

Cysteine alkylation was set as a fixed modification. Oxidation on Met was specified as a variable modification. The quantitative method was set to TMT-6plex, and the false discovery rate for protein identification and PSM identification was set to $1 \%$.

\section{Protein Annotation}

The GO annotation proteome was derived from the UniProt-GOA database (www. http://www.ebi.ac.uk/GOA/). Subsequently, proteins were classified according to GO annotation on the basis of three categories: biological process, cellular component, and molecular function. The KEGG database was used to annotate protein pathways. KEGG online service tools KAAS (http://www.genome.jp/kaas-bin/kaas_main) were used to annotate the KEGG database descriptions of proteins, and then the annotation results were mapped on the KEGG pathway database by using the KEGG online service tool KEGG mapper (http://www.kegg.jp/kegg/mapper.html). For domain annotation, the InterProScan database (http://www.ebi.ac.uk/interpro/) was used to annotate the domain functional descriptions of identified proteins. For subcellular localization, WoLFSPORT (a subcellular localization predication software, http://www.genscript.com/psort/wolf_psort.html) was used. WoLFSPORT is an updated version of PSORT/PSORT II for the prediction of eukaryotic sequences.

\section{Statistical Analysis}

For GO enrichment and pathway analysis, a two-tailed Fisher's exact test was used to test the enrichment of the DEPs against all identified proteins. A GO term or KEGG pathway with a Pvalue $<0.05$ was considered significant. The proteins with TMT intensity values were considered quantified, and the minimal PIF was set as 0.75 . Statistical analyses were carried out

Peer) reviewing PDF | (2019:04:36887:2:0:NEW 17 Sep 2019) 
190

191

192

193

194

195

196

197

198

199

200

201

202

203

204

205

206

207

208

209

210

211

212

213

214

215

216

217

218

219

220

221

222

223

224

225

226

227

228

in SPSS ver. 19.0 (SPSS Inc. Chicago, US). All reported values represent the averages of three replicates with the standard deviation (mean $\pm \mathrm{SD}$ ).

\section{Quantitative real-time PCR validation}

Total RNA was extracted using a Ultrapure RNA Kit according to the manufacturer's protocol (Code: CW0597, CWBIO, Beijing, China). First-strand cDNA synthesis was carried out using a SuperScript ${ }^{\mathrm{TM}}$ IV First-Strand Synthesis System according to the manufacturer's protocol (Code:18091050, ThermoFisher, Massachusetts, USA). QRT-PCR was performed on ABI 7500 Real-Time PCR System (Roche, Basel, Switzerland) using UltraSYBR Mixture (High ROX) Kit (Code: CW2602, CWBIO, Beijing, China) with the primers listed in Table S1. The CaACTIN (Capana12g001934) was used as an internal standard to calculate relative folddifferences based on comparative cycle threshold $(2-\Delta \Delta \mathrm{Ct})$ values.

\section{Results}

\section{Primary MS Data and Quantitative Proteome Analysis}

A total of 252,213 secondary spectra were obtained by MS analysis of the mock treated and Se treated pepper seedlings. A Pearson correlation coefficient analysis indicated high replicability of the experiment (Fig.1A). A search of the proteome Capsicum annuum database indicated 47,316 spectra were available, and the utilization rate of the spectra was $18.8 \%$. A total of 24,048 peptide fragments were identified by spectral analysis, including 21,704 unique peptide fragments (Fig.1B). Most of the peptides contained 7-20 amino acids, results consistent with the trypsin enzymatic hydrolysis and high-energy collision dissociation fragmentation (Fig.1C). The molecular weights of the proteins were negatively correlated with their coverage (Fig.1D). The first-order mass error of most spectra was less than $10 \mathrm{ppm}$, in agreement with the high accuracy of orbital well MS. The results indicated high mass accuracy of the MS data (Fig.1E). Principal component analysis of the quantitative protein data for all samples is presented in Fig.1F. Detailed information on the identified peptides, including amino acid sequences, protein descriptions, carried charge of peptide, The maximal posterior error probability for peptides(PEP), is listed in Table S2.

A total of 4,693 proteins were identified, among which 3,938 were quantifiable. To understand the functions and characteristics of the proteins identified and quantified in the data, we performed detailed annotation analysis on the basis of Gene Ontology (GO), protein domain, Kyoto Encyclopedia of Genes and Genomes (KEGG) pathway, KOG functional classification (eukaryotic orthologous groups), and subcellular localization (Table S3).

\section{Effects of Se Treatment on the Global Proteome of Pepper Seedlings}

A total of 200 DEPs were identified with a fold-difference expression threshold of 1.5 (Se/Mock ratio $\geq 1.5$ or $\leq 0.667$ ) and a t-test P-value $<0.05$ (Table S4). All identified proteins and DEPs under Se treatment were grouped into three GO categories (biological process, cellular component, and molecular function) (Fig.2A). In the biological process category, 1,594 
229 identified proteins and 67 DEPs were involved in 'metabolic processes,' 1,423 identified proteins 230 and 46 DEPs were involved in 'cellular processes,' and 1,107 identified proteins and 46 DEPs 231 were involved in 'single-organism processes.' In the molecular function category, 1,959 232 identified proteins and 74 DEPs had 'catalytic activities,' 1,899 identified proteins and 74 DEPs 233 had 'binding activities,' and 178 identified proteins and one DEP had 'structural activities.' In the 234 cellular components category, 737 identified proteins and 10 DEPs were 'cell'-related proteins; 235418 identified proteins and three DEPs were 'macromolecular complex'-related proteins; and 236402 identified proteins and four DEPs were 'organelle'-related proteins. The distribution of the 237 GO annotations of the up-regulated and down-regulated DEPs is shown in Fig.S1. We also used 238 WoLFPSORT (http://wolfpsort.org/) software to determine the subcellular location prediction 239 and classification statistics for all identified proteins and DEPs (Fig.2B), which were grouped 240 according to their subcellular localizations. All identified proteins were classified into 15 241 subcellular components, including 1,728 chloroplast-localized proteins, 1,283 cytoplasm242 localized proteins, and 784 nuclear-localized proteins. For the DEPs, 13 subcellular components 243 were identified, including 72 cytoplasm-localized DEPs, 44 chloroplast-localized DEPs, and 42 244 nuclear-localized DEPs.

245 The expression profiles of the DEPs in six samples are presented in a heat map (Fig.3A). To 246 reveal the changing trends among the six samples, we assigned all DEPs to one of three clusters 247 (I-III) by using MeV (http://www.tm4.org/ mev.html) with the K-means method (Fig.3B). The 248 proteins in clusters I and II showed up-regulation, whereas the proteins in cluster III were down249 regulated in the Se stress treatment replicates. Among the DEPs, 172 were up-regulated and 28 250 were down-regulated (Fig. 3c and 3d). The $16.6 \mathrm{kDa}$ heat shock protein (HSP) (A0A1U8FR15) 251 and two $18.5 \mathrm{kDa}$ class I HSPs (A0A1U8DWR1, A0A1U8E6M6) were up-regulated over nine252 fold by Se treatment compared with the mock treatment. In addition, a glycine-rich protein 253 (A0A2G2ZTC5) and histone H1 protein were down-regulated more than two-fold by Se

254

255

256

257

258

259

260

261

262

263

264

265

266

267

treatment compared with the mock treatment. A total of 136 DEPs were classified into $20 \mathrm{KOG}$ terms. 'Post-translational modification, protein turnover, chaperones' contained the largest DEPs (Fig.4).

\section{Enrichment Analysis of DEPs Under Se Treatment}

To determine whether the DEPs were significantly enriched in some functional types, we performed an enrichment analysis of differentially expressed proteins by using GO classification, KEGG pathways, and protein domains. Among the DEPs, most up-regulated proteins were enriched in 'sequence-specific DNA binding,' 'iron ion binding,' 'prephenate dehydrogenase $\left(\mathrm{NAD}^{+} / \mathrm{NADP}^{+}\right.$) activity,' 'nucleic acid binding transcription factor activity,' and 'apoplast' (Fig. 5A). For the down-regulated proteins, the top five enriched GO terms were 'thiamine-containing compound metabolic process,' 'chlorophyll metabolic process,' 'porphyrin-containing compound biosynthetic process,' 'water-soluble vitamin biosynthetic process,' and 'vitamin biosynthetic process' (Fig.5B).

Peer) reviewing PDF | (2019:04:36887:2:0:NEW 17 Sep 2019) 
268

269

270

271

272

273

274

275

276

277

278

279

280

281

282

283

284

285

286

287

288

289

290

291

292

293

294

295

296

297

298

299

300

301

302

303

304

305

306

Under Se treatment, 131 DEPs were grouped into different KEGG pathways, seven of which were enriched $(\mathrm{P}<0.05)$. For the up-regulated proteins, the DEPs were associated with 'protein processing in endoplasmic reticulum,' 'endocytosis,' 'sesquiterpenoid and triterpenoid biosynthesis,' 'SNARE interactions in vesicular transport,' and 'plant-pathogen interaction' (Fig. 6A). For the down-regulated proteins, the DEPs were associated with 'thiamine metabolism' and 'porphyrin and chlorophyll metabolism' (Fig.6B). In the present study, 32 DEPs in the Se treated pepper were identified to be involved in nine metabolic pathways, most of which were significantly up-regulated by Se treatment. However, in the 'thiamine metabolism' pathway, four proteins (A0A2G3ALC4, A0A1U8FC73, A0A2G3A131, and A0A2G2Z8I0) were significantly down-regulated by Se treatment (Table 1).

Protein domain enrichment analysis revealed that 25 protein domains were enriched in the DEPs (Fig. 7A). The five most enriched protein domains were 'alpha crystallin/Hsp20 domain' (13 proteins), 'HSP20-like chaperone' (14 proteins), 'target SNARE coiled-coil homology domain' (four proteins), 'heat shock protein $70 \mathrm{kDa}$ ' (five proteins), and 'DnaJ domain' (six proteins). Many HSPs were identified according to the results of the protein domain enrichment analysis. In total, 23 HSPs were significantly up-regulated (Fig.7B). The expression levels of some HSP genes were basically consistent with the proteomic analyses (Fig.S2)

\section{Discussion}

With the increased accumulation of Se in the soil as a result of anthropogenic activity, Se, which has toxic effects on plants at certain concentrations, has gradually become a potential environmental risk factor. In studies of Se, quantitative proteomics analyzed through MS have been applied primarily in analysis of bio-transformation of Se-containing compounds in experimental models such as animals, yeast, and cancer cells (Zhang et al., 2010; Sinha et al., 2016). In general, proteomic analysis technologies have rarely been applied in the study of Se metabolism in plants. In the present study, we conducted a TMT-based quantitative proteomic analysis of the responses of pepper shoots to Se stress. Many DEPs were identified, and a group of proteins potentially involved in Se stress responses were identified. The Se-responsive DEPs and the associated metabolic pathways may play critical roles in Se stress signaling and responses in pepper.

Se is chemically similar to sulfur and is absorbed by plants through similar metabolic pathways (Van Hoewyk et al., 2008; Cakir, Turgut-Kara\& Ari, 2016). Most plants nonspecifically absorb Se from the environment through sulfate transporters and assimilate Se into organic forms of Se through the S metabolic pathway (Cappa et al., 2014). To date, studies on Se-tolerance mechanisms have focused on some Se-hyperaccumulating plants (Freeman et al., 2010; Sabbagh \& Van Hoewyk 2012; Cappa et al., 2015). In Cardamine hupingshanensis seedlings, the expression of the sulfite oxidase ( $S O X$ ) gene in the roots is up-regulated after the addition of selenite, thus indicating that selenite may first be converted to selenate, and then the selenate is metabolized (Zhou et al., 2018). 
307

308

309

310

311

312

313

314

315

316

317

318

319

320

321

322

323

324

325

326

327

328

329

330

331

332

333

334

335

336

337

338

339

340

341

342

343

344

345

346

Plants have evolved several efficient and complex strategies for dealing with different abiotic stresses, including Se stress (Chen et al., 2002; Lyons, Stangoulis \& Graham,2004; PerezClemente et al., 2013; Schiavon et al., 2013). GO analysis revealed that 14 proteins were associated with 'response to stimulus,' including 11 up- and three down-regulated proteins significantly altered by Se stress in pepper. Among the proteins, a peroxidase (A0A1U8DW23) and a glutathione peroxidase (A0A2G3AE16) were up-regulated over 1.5-fold by Se treatment, thus suggesting that reactive oxygen species accumulate after Se treatment. In the present study, the expression of two pathogenesis-related proteins (STH-21, A0A1U8GEH7; PR4, I6VW44) significantly decreased. Pathogenesis-related proteins are a class of stress-tolerant proteins that are promising tools for plant genetic engineering (Ali et al., 2018). KOG analysis revealed that 36 proteins were related to the term 'posttranslational modification, protein turnover, chaperones'. The identified DEPs may be involved in plant responses to Se stress. In pepper, nine 'amino acid transport and metabolism'-related proteins and ten 'secondary metabolites biosynthesis, transport and catabolism'-associated proteins were identified. The changes in the DEPs suggested that Se stress may influence metabolite content in pepper.

Plants are thought to produce specific metabolites in response to Se stress (Fernandes et al., 2018; Pilon-Smits et al 2009). Transcriptomic analysis of Se-treated Arabidopsis thaliana has revealed that sulfur content decreases while the expression of sulfur absorption and metabolism genes increases, and the signaling pathways for ethylene and jasmonic acid respond to Se stress (Van Hoewyk et al., 2008). Small RNA and degradome sequencing analysis of Astragalus chyrsochlorus callus has revealed that miR167a, miR319, miR1507a, miR4346, miR7767-3p, miR7800, miR9748, and miR-n93 target transcription factors, disease resistance proteins, cysteine synthase, plant hormone signal transduction, plant-pathogen interaction, and sulfur metabolism pathways in response to Se stimuli (Cakir, Candar-Cakir \& Zhang, 2016). In the present study, KEGG enrichment analysis revealed that seven pathways, including the sesquiterpenoid and triterpenoid biosynthesis pathway, plant-pathogen interaction, and thiamine metabolism pathway, were enriched after Se treatment. In addition, 32 DEPs involved in nine metabolic pathways were identified. Nine up-regulated DEPs were associated with cysteine and methionine metabolism. The main cause of plant Se poisoning is thought to be the incorrect synthesis of selenomethionine and selenocysteine (SeCys) into proteins, thereby causing changes or instability in the structures of proteins (Sabbagh \& Van Hoewyk 2012; Van Hoewyk, 2013).High concentrations of SeCys in cells would lead to Se poisoning, and SeCys transformation is a direct means of Se detoxification (Pilon et al, 2003; Tamaoki, Freeman \& Pilon-Smits,2008; Sabbagh \& Van Hoewyk 2012). Terpenoids, the most diverse class of chemicals produced by plants, are involved in protection against various abiotic factors (Lange, 2015; Tholl, 2015). For example, terpenoids are considered to be important defensive metabolites in Eucalyptus froggattii seedlings (Goodger, Heskes \& Woodrow, 2013).Four upregulated DEPs were associated with 'ubiquinone and other terpenoid-quinone biosynthesis'and 'sesquiterpenoid and triterpenoid biosynthesis,' thus indicating that Se stress influenced the accumulation of some terpenoids. 
HSPs were initially defined as proteins rapidly up-regulated by heat stress (Hartl \& HayerHartl, 2002). Studies increasingly show that HSP concentrations in plants increase rapidly as environmental conditions deteriorate (Murakami et al., 2004; Hu, Hu \& Han, 2009; Lee, Yun \& Kwon, 2012). HSPs are a class of evolutionarily conserved proteins that can be divided into five families according to molecular weight and sequence homology: small HSPs (molecular weight from $15 \mathrm{kDa}$ to $42 \mathrm{kDa}$ ), HSP60, HSP70, HSP90, and HSP100 (Boston, Viitanen \& Vierling, 1996; Wang et al., 2004; Waters, 2013). HSP90 and HSP70 are essential for plant resistance to pathogen infections (Kanzaki et al., 2003; Noel., 2007; Chen et al., 2010). AtHSP17.6A is induced by osmotic stress, and PtHSP17.8 is involved in tolerance to heat and salt stress (Sun et al., 2001; Li et al., 2016). Among the Se-stress-induced DEPs in pepper, several HSPs were identified. A total of 14 HSP20, four HSP40, and five HSP70 proteins were significantly upregulated by salt stress, thus suggesting molecular cross-talk between heat shock responses and Se stress.

\section{Conclusions}

A TMT-based proteomic method was used to investigate changes in protein levels between control and Se treated pepper seedlings. In total, 4,693 proteins and 200 DEPs were identified. A number of DEPs were found to be mainly involved in responses to stress and metabolic processes. Our results provide basic tools for identifying candidate proteins and the molecular mechanisms of the Se stress response in pepper plants.

\section{ADDITIONAL INFORMATION AND DECLARATIONS}

\section{Acknowledgements}

We are grateful to the PTM Biolabs company for technical support. We thank International Science Editing ( http://www.internationalscienceediting.com ) for editing this manuscript. Funding

This work was funded by the Natural Science Foundation of Zhejiang Province, China, Grant No. LQ17C150003. The National Natural Science Foundation of China, Grant No. 31701967, the key research and development program of Zhejiang Province, China, Grant No. 2017C02018 and the key research and development program of Hangzhou, China, Grant No. 20180416A07. The funders had no role in study design, data collection and analysis, decision to publish, or preparation of the manuscript.

\section{Grant Disclosures}

The following grant information was disclosed by the authors:

the Natural Science Foundation of Zhejiang Province:LQ17C150003 
382 National Natural Science Foundation of China: 31701967

383 Key research and development program of Zhejiang Province, China: 2017C02018

384 Key research and development program of Hangzhou, China: 20180416A07

385 Competing Interests

386 The authors declare there are no competing interests.

387

388

389

390

391

392

393

394

395

396

397

398

399

400

401

402

403

404

405

406

407

408

409

410

411

412

413

414

415

\section{Author Contributions}

- Chenghao Zhang, Chengwu Jin and Chenliang Yu conceived and designed the experiments, contributed reagents/materials/analysis tools, analyzed the data, prepared figures and/or tables, authored or reviewed drafts of the paper.

-Baoyu Xu, Wei Geng and Yunde Shen, contributed reagents/materials/analysis tools, performed the experiments, analyzed the data prepared figures and/or tables

-Dongji Xuan,Qixian Lai, and Chenjia Shen, analyzed the data, authored or reviewed drafts of the paper, approved the final draft.

\section{Data Availability}

Data are available via ProteomeXchange with identifier

PXD013257(http://www.ebi.ac.uk/pride/archive/projects/PXD013257). FTP Download:

ftp://ftp.pride.ebi.ac.uk/pride/data/archive/2019/09/PXD013257.

\section{References}

Aggarwal M, Sharma S, Kaur N, Pathania D, Bhandhari K, Kaushal N, Kaur R, Singh K, Srivastava A, and Nayyar H. 2011. Exogenous Proline Application Reduces Phytotoxic Effects of Selenium by Minimising Oxidative stress and Improves Growth in Bean ( Phaseolus vulgaris L.) Seedlings. Biological Trace Element Research 140:354-367.

Ali S, Ganai BA, Kamili AN, Bhat AA, Mir ZA, Bhat JA, Tyagi A, Islam ST, Mushtaq M, Yadav P,Rawat S, and Grover A. 2018. Pathogenesis-related proteins and peptides as promising tools for engineering plants with multiple stress tolerance. Microbiol Res 212213:29-37.

Boston RS, Viitanen PV, and Vierling E. 1996. Molecular chaperones and protein folding in plants. Plant Molecular Biology 32:191-222.

Cakir O, Candar-Cakir B, and Zhang B. 2016. Small RNA and degradome sequencing reveals important microRNA function in Astragalus chrysochlorus response to selenium stimuli. Plant Biotechnol J 14:543-556.

Cakir O, Turgut-Kara N, and Ari S. 2016. Selenium induced selenocysteine methyltransferase gene expression and antioxidant enzyme activities in Astragalus chrysochlorus. Acta Botanica Croatica 75:11-16. 
416 Cappa JJ, Cappa PJ, El Mehdawi AF, Mcaleer JM, Simmons MP, and Pilon-Smits EA. 2014.

417

418

419

420

421

422

423

424

425

426

427

428

429

430

431

432

433

434

435

436

437

438

439

440

441

442

443

444

445

446

447

448

449

450

451

452

453

Characterization of selenium and sulfur accumulation across the genus Stanleya (Brassicaceae): A field survey and common-garden experiment. American Journal of Botany 101:830-839.

Cappa JJ, Yetter C, Fakra S, Cappa PJ, DeTar R, Landes C, Pilon-Smits EAH, and Simmons MP. 2015. Evolution of selenium hyperaccumulation in Stanleya (Brassicaceae) as inferred from phylogeny, physiology and X-ray microprobe analysis. New Phytologist 205:583595.

Chen LC, Yang FM, Xu J, Hu Y, Hu QH, Zhang YL, and Pan GX. 2002. Determination of selenium concentration of rice in China and effect of fertilization of selenite and selenate on selenium content of rice. J Agric Food Chem 50:5128-5130.

Chen LT, Hamada S, Fujiwara M, Zhu TH, Thao NP, Wong HL, Krishna P, Ueda T, Kaku H, Shibuya N, Kawasaki T, and Shimamoto, K. 2010. The Hop/Sti1-Hsp90 Chaperone Complex Facilitates the Maturation and Transport of a PAMP Receptor in Rice Innate Immunity. Cell Host \& Microbe 7:185-196.

Choi GS, Kim JH, Lee DH, Kim JS, and Ryu KH. 2005. Occurrence and Distribution of Viruses Infecting Pepper in Korea.

Chu J, Yao X, and Zhang Z. 2010. Responses of Wheat Seedlings to Exogenous Selenium Supply Under Cold Stress. Biological Trace Element Research 136:355-363.

Djanaguiraman M, Prasad PVV, and Seppanen M. 2010. Selenium protects sorghum leaves from oxidative damage under high temperature stress by enhancing antioxidant defense system. Plant Physiology \& Biochemistry Ppb 48:999-1007.

El Kassis E, Cathala N, Rouached H, Fourcroy P, Berthomieu P, Terry N, and Davidian JC. 2007. Characterization of a selenate-resistant Arabidopsis mutant. Root growth as a potential target for selenate toxicity. Plant Physiology 143:1231-1241.

El-Ramady HR, Domokos-Szabolcsy É, Shalaby TA, Prokisch J, and Fári M. 2015. Selenium in Agriculture: Water, Air, Soil, Plants, Food, Animals and Nanoselenium.

Elrashidi MA, Adriano DC, Workman SM, and Lindsay WL. 1987. Chemical-Equilibria Of Selenium In Soils - a Theoretical Development. Soil Science 144:141-152.

Fernandes J, Hu X, Smith MR, Go YM, and Jones DP. 2018. Selenium at the redox interface of the genome, metabolome and exposome. Free Radical Biology And Medicine 127:215227.

Freeman JL, Tamaoki M, Stushnoff C, Quinn CF, Cappa JJ, Devonshire J, Fakra SC, Marcus MA, McGrath SP, Van Hoewyk D, and Pilon-Smits, EAH. 2010. Molecular Mechanisms of Selenium Tolerance and Hyperaccumulation in Stanleya pinnata. Plant Physiology 153:1630-1652.

Galinha C, Sanchez-Martinez M, Pacheco AMG, Freitas MD, Coutinho J, Macas B, Almeida AS, Perez-Corona MT, Madrid Y, and Wolterbeek HT. 2015. Characterization of selenium-

PeerJ reviewing PDF | (2019:04:36887:2:0:NEW 17 Sep 2019) 
454

455

456

457

458

459

460

461

462

463

464

465

466

467

468

469

470

471

472

473

474

475

476

477

478

479

480

481

482

483

484

485

486

487

488

489

490

491

enriched wheat by agronomic biofortification. Journal Of Food Science And TechnologyMysore 52:4236-4245.

Goodger JQD, Heskes AM, and Woodrow IE. 2013. Contrasting ontogenetic trajectories for phenolic and terpenoid defences in Eucalyptus froggattii. Annals Of Botany 112:651-659.

Hao J, Guo H, Shi XN, Wang Y, Wan QH, Song YB, Zhang L, Dong M, and Shen CJ. 2017. Comparative proteomic analyses of two Taxus species (Taxus $\mathrm{X}$ media and Taxus mairei) reveals variations in the metabolisms associated with paclitaxel and other metabolites. Plant and Cell Physiology 58:1878-1890.

Hartikainen H, Ekholm P, Piironen V, Xue T, Koivu T, and Yli-Halla M. 1997. Quality of the ryegrass and lettuce yields as affected by selenium fertilization. Agricultural \& Food Science in Finland 6:381-387.

Hartl FU, and Hayer-Hartl M. 2002. Protein folding - Molecular chaperones in the cytosol: from nascent chain to folded protein. Science 295:1852-1858.

Hladun KR, Parker DR, Tran KD, and Trumble JT. 2013. Effects of selenium accumulation on phytotoxicity, herbivory, and pollination ecology in radish (Raphanus sativus L.). Environmental Pollution 172:70-75.

$\mathrm{Hu}$ WH, Hu GC, and Han B. 2009. Genome-wide survey and expression profiling of heat shock proteins and heat shock factors revealed overlapped and stress specific response under abiotic stresses in rice. Plant Science 176:583-590.

Jia M, Zhang Y, Huang B, and Zhang H. 2019. Source apportionment of selenium and influence factors on its bioavailability in intensively managed greenhouse soil: A case study in the east bank of the Dianchi Lake, China. Ecotoxicology And Environmental Safety 170:238245.

Kanzaki H, Saitoh H, Ito A, Fujisawa S, Kamoun S, Katou S, Yoshioka H, and Terauchi R. 2003. Cytosolic HSP90 and HSP70 are essential components of INF1-mediated hypersensitive response and non-host resistance to Pseudomonas cichorii in Nicotiana benthamiana. Molecular Plant Pathology 4:383-391.

Kong LA, Wang M, and Bi DL. 2005. Selenium modulates the activities of antioxidant enzymes, osmotic homeostasis and promotes the growth of sorrel seedlings under salt stress. Plant Growth Regulation 45:155-163.

Kumar M, Bijo AJ, Baghel RS, Reddy CRK, and Jha B. 2012. Selenium and spermine alleviate cadmium induced toxicity in the red seaweed Gracilaria dura by regulating antioxidants and DNA methylation. Plant Physiology And Biochemistry 51:129-138.

Kuppusamy S, Yoon YE, Song YK, Kim JH, Kim HT, and Yong BL. 2017. Does long-term application of fertilizers enhance the micronutrient density in soil and crop?-Evidence from a field trial conducted on a 47-year-old rice paddy. Journal of Soils \& Sediments:114.

Lange BM. 2015. The Evolution of Plant Secretory Structures and Emergence of Terpenoid 
493

494

495

496

497

498

499

500

501

502

503

504

505

506

507

508

509

510

511

512

513

514

515

516

517

518

519

520

521

522

523

524

525

526

527

Lee JH, Yun HS, and Kwon C. 2012. Molecular communications between plant heat shock responses and disease resistance. Molecules And Cells 34:109-116.

Li JB, Zhang J, Jia HX, Li Y, Xu XD, Wang LJ, and Lu MZ. 2016. The Populus trichocarpa PtHSP17.8 involved in heat and salt stress tolerances. Plant Cell Reports 35:1587-1599.

Lyons GH, Stangoulis JCR, and Graham RD. 2004. Exploiting micronutrient interaction to optimize biofortification programs: The case for inclusion of selenium and iodine in the HarvestPlus program. Nutrition Reviews 62:247-252.

Molnárová M, and Fargašová A. 2009. Se (IV) phytotoxicity for monocotyledonae cereals ( Hordeum vulgare L., Triticum aestivum L.) and dicotyledonae crops ( Sinapis alba L., Brassica napus L.). Journal of Hazardous Materials 172:854-861.

Noel LD, Cagna G, Stuttmann J, Wirthmuller L, Betsuyaku S, Witte CP, Bhat R, Pochon N, Colby T, and Parker JE. 2007. Interaction between SGT1 and Cytosolic/Nuclear HSC70 chaperones regulates Arabidopsis immune responses. Plant Cell 19:4061-4076.

Paciolla C, De Leonardis S, and Dipierro S. 2011. Effects of selenite and selenate on the antioxidant systems in Senecio scandens L. Plant Biosystems 145:253-259.

Perez-Clemente RM, Vives V, Zandalinas SI, Lopez-Climent MF, Munoz V, and GomezCadenas A. 2013. Biotechnological Approaches to Study Plant Responses to Stress. Biomed Research International.

Pilon M, Owen JD, Garifullina GF, Kurihara T, Mihara H, Esaki N, and Pilon-Smits EAH. 2003. Enhanced selenium tolerance and accumulation in transgenic Arabidopsis expressing a mouse selenocysteine lyase. Plant Physiology 131:1250-1257.

Pilon-Smits EAH, Quinn CF, Tapken W, Malagoli M, and Schiavon M. 2009. Physiological functions of beneficial elements. Current Opinion In Plant Biology 12:267-274.

Pukacka S, Ratajczak E, and Kalemba E. 2011. The protective role of selenium in recalcitrant Acer saccharium L. seeds subjected to desiccation. Journal Of Plant Physiology 168:220225.

Quinn CF, Freeman JL, Galeas ML, Klamper EM, and Pilon-Smits EAH. 2008. The role of selenium in protecting plants against prairie dog herbivory: implications for the evolution of selenium hyperaccumulation. Oecologia 155:267-275.

Sabbagh M, and Van Hoewyk D. 2012. Malformed Selenoproteins Are Removed by the Ubiquitin-Proteasome Pathway in Stanleya pinnata. Plant and Cell Physiology 53:555564.

Sager M. 2006. Selenium in agriculture, food, and nutrition. Pure And Applied Chemistry 78:111-133.

Schiavon M, Dall'Acqua S, Mietto A, Pilon-Smits EAH, Sambo P, Masi A, and Malagoli M.

Peer) reviewing PDF | (2019:04:36887:2:0:NEW 17 Sep 2019) 
528

529

530

531

532

533

534

535

536

537

538

539

540

541

542

543

544

545

546

547

548

549

550

551

552

553

554

555

556

557

558

559

560

561

562

563

564

2013. Selenium Fertilization Alters the Chemical Composition and Antioxidant Constituents of Tomato (Solanum lycopersicon L.). J Agric Food Chem 61:10542-10554.

Semnani S, Roshandel G, Zendehbad A, Keshtkar A, Rahimzadeh H, Abdolahi N, Besharat S, Moradi A, Mirkarimi H, and Hasheminasab S. 2010. Soils selenium level and esophageal cancer: An ecological study in a high risk area for esophageal cancer. Journal of Trace Elements in Medicine \& Biology 24:174-177.

Shi HZ, Ishitani M, Kim CS, and Zhu JK. 2000. The Arabidopsis thaliana salt tolerance gene $\mathrm{SOS} 1$ encodes a putative $\mathrm{Na}+\mathrm{H}+$ antiporter. Proceedings Of the National Academy Of Sciences Of the United States Of America 97:6896-6901.

Shibagaki N, Rose A, McDermott JP, Fujiwara T, Hayashi H, Yoneyama T, and Davies JP. 2002. Selenate-resistant mutants of Arabidopsis thaliana identify Sultr1;2, a sulfate transporter required for efficient transport of sulfate into roots. Plant Journal 29:475-486.

Sinha I, Karagoz K, Fogle RL, Hollenbeak CS, Zea AH, Arga KY, Stanley AE, Hawkes WC, and Sinha R. 2016. "Omics" of Selenium Biology: A Prospective Study of Plasma Proteome Network Before and After Selenized-Yeast Supplementation in Healthy Men. Omics-a Journal Of Integrative Biology 20:202-213.

Song ZP, Shao HF, Huang HG, Shen Y, Wang LZ, Wu FY, Han D, Song JY, and Jia HF. 2017. Overexpression of the phosphate transporter gene OsPT8 improves the Pi and selenium contents in Nicotiana tabacum. Environmental And Experimental Botany 137:158-165.

Sun WN, Bernard C, van de Cotte B, Van Montagu M, and Verbruggen N. 2001. At-HSP17.6A, encoding a small heat-shock protein in Arabidopsis, can enhance osmotolerance upon overexpression. Plant Journal 27:407-415.

Tamaoki M, Freeman JL, and Pilon-Smits EAH. 2008. Cooperative ethylene and jasmonic acid signaling regulates selenite resistance in Arabidopsis. Plant Physiology 146:1219-1230.

Thavarajah D, Ruszkowski J, and Vandenberg A. 2008. High potential for selenium biofortification of lentils ( Lens culinaris L.). J Agric Food Chem 56:10747-10753.

Tholl D. 2015. Biosynthesis and Biological Functions of Terpenoids in Plants. Advances in Biochemical Engineering/biotechnology 148:63.

Van Hoewyk D. 2013. A tale of two toxicities: malformed selenoproteins and oxidative stress both contribute to selenium stress in plants. Annals Of Botany 112:965-972.

Van Hoewyk D, Takahashi H, Inoue E, Hess A, Tamaoki M, and Pilon-Smits EA. 2008. Transcriptome analyses give insights into selenium-stress responses and selenium tolerance mechanisms in Arabidopsis. Physiol Plant 132:236-253.

Wang CQ. 2011. Water-stress mitigation by selenium in Trifolium repens L. Journal of Plant Nutrition and Soil Science 174:276-282.

Wang WX, Vinocur B, Shoseyov O, and Altman A. 2004. Role of plant heat-shock proteins and molecular chaperones in the abiotic stress response. Trends In Plant Science 9:244-252.

PeerJ reviewing PDF | (2019:04:36887:2:0:NEW 17 Sep 2019) 
565

566

567

568

569

570

571

572

573

574

575

576

577

578

579

580

581

582

583

584

585

586

587

588

589

590

591

592

593

594

595

596

597

598

599

600

601

Wang YD, Wang X, and Wong YS. 2012. Proteomics analysis reveals multiple regulatory mechanisms in response to selenium in rice. Journal Of Proteomics 75:1849-1866.

Waters ER. 2013. The evolution, function, structure, and expression of the plant sHSPs. Journal Of Experimental Botany 64:391-403.

White PJ. 2016. Selenium accumulation by plants. Annals Of Botany 117:217-235.

Xu DB, Yuan HW, Tong YF, Zhao L, Qiu LL, Guo WB, Shen CJ, Liu HJ, Yan DL, and Zheng BS. 2017. Comparative Proteomic Analysis of the Graft Unions in Hickory (Carya cathayensis) Provides Insights into Response Mechanisms to Grafting Process. Frontiers In Plant Science 8.

Zhang JH, Wang L, Anderson LB, Witthuhn B, Xu YJ, and Lu JX. 2010. Proteomic Profiling of Potential Molecular Targets of Methyl-Selenium Compounds in the Transgenic Adenocarcinoma of Mouse Prostate Model. Cancer Prevention Research 3:994-1006.

Zhang LH, Hu B, Li W, Che RH, Deng K, Li H, Yu FY, Ling HQ, Li YJ, and Chu CC. 2014. OsPT2, a phosphate transporter, is involved in the active uptake of selenite in rice. New Phytologist 201:1183-1191.

Figure legends

Figure1. Quality control (QC) validation of Mass spectrometer (MS) data. (A) Heatmap of Pearson correlation coefficients from all quantified proteins between each pair of samples. Protein were extracted in three biological replicates for each sample group. All protein samples were trypsin digested and analyzed by HPLC-MS/MS. (B) Two-dimensional scatter plot of PCA (principal component analysis) distribution of all samples using quantified proteins. (C) Relationship between molecular weight and coverage of proteins identified by mass spectrometry. (D) Mass error distribution of all identified peptides. (E) Basic statistical data of MS results. (F) Length distribution of all identified phosphorylated peptides.

Figure 2. Classification of all identified proteins and DEPs. (A) GO analysis of all identified proteins and DEPs. All proteins were classified by GO terms based on three categories: molecular function, biological process and cellular component. (B) Subcellular classify of all identified proteins and DEPs.

Figure 3. Impacts Se stress treatment on proteome levels in pepper. (A) Expression profiles of the DEPs response to Se stress. (B-D) All DEPs were analyzed and clustered into three major Clusters by K-means method. (E) Volcano plot of DEPs. (F). The numbers of upand down-regulated proteins in the Se treatment seedlings compared to the mock seedlings. 
602

603 Figure 4. KOG functional classification chart of differentially expressed proteins.

604

605

606

Figure 5. GO enrichment analysis of DEPs. Distribution of the up-regulated (A) and downregulated (B) proteins with $\mathrm{GO}$ enrichment analysis.

607

608

609

Figure 6. KEGG enrichment analysis of the DEPs in pepper after Se stress treatment. (A) Significantly enriched KEGG terms of the up-regulated proteins. (B) Significantly

610 enriched KEGG terms of the down-regulated proteins.

611

612

613

Figure 7. Domain enrichment analysis of the DEPs in pepper after Se stress treatment. (A)

614 Protein domain enrichment bubble plot of differentially expressed proteins. (B) The accumulation of HSP proteins after Se stress treatment.

615 


\section{Figure 1}

Quality control (QC) validation of Mass spectrometer (MS) data.

(A) Heatmap of Pearson correlation coefficients from all quantified proteins between each pair of samples. Protein were extracted in three biological replicates for each sample group. All protein samples were trypsin digested and analyzed by HPLC-MS/MS. (B) Two-dimensional scatter plot of PCA (principal component analysis) distribution of all samples using quantified proteins. (C) Relationship between molecular weight and coverage of proteins identified by mass spectrometry. (D) Mass error distribution of all identified peptides. (E) Basic statistical data of MS results. (F) Length distribution of all identified phosphorylated peptides. 


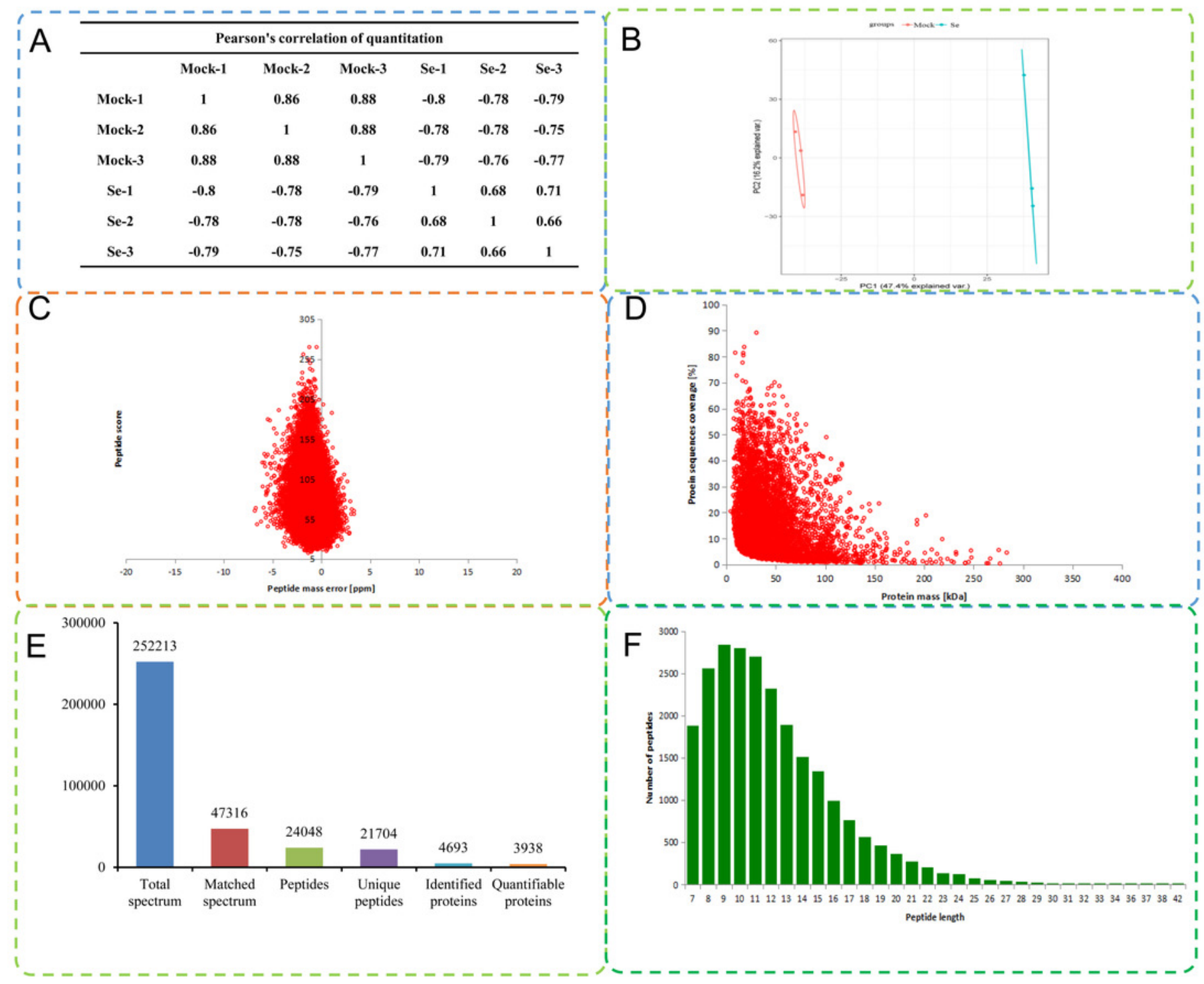


Figure 2

Classification of all identified proteins and DEPs.

(A) GO analysis of all identified proteins and DEPs. All proteins were classified by GO terms

based on three categories: molecular function, biological process and cellular component. (B)

Subcellular classify of all identified proteins and DEPs.

A

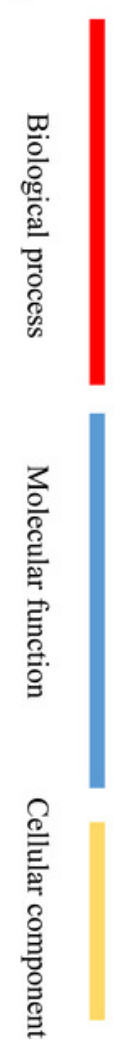

cellular component organization or biogenesi

biological regulation

biological regulation
multi-organism process

cellular process

multicellular organismat process

multicellular organismal process

localization

response to stimulus

single-organism proces

metabolic proces

reproduction

developmental process

transporter activity

signal transducer activity

structural molecule activity transcription factor activity, protein binding

molecular function regulator

nutrient reservoir activity

antioxidant activity

nucleic acid binding transcription factor activity

binding

catalytic activity

molecular transducer activity

electron carrier activity

extracellular regio

supramolecular complex

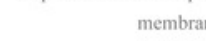

macromolecular complex

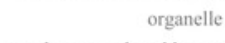

membrane-enclosed lume

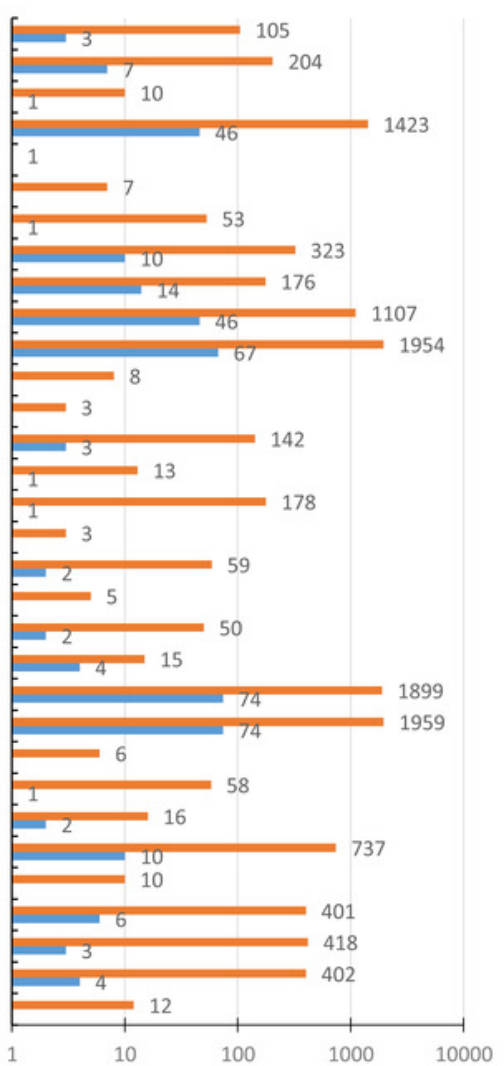

m Identified $\mathbf{m}$ Se vs Mock
B

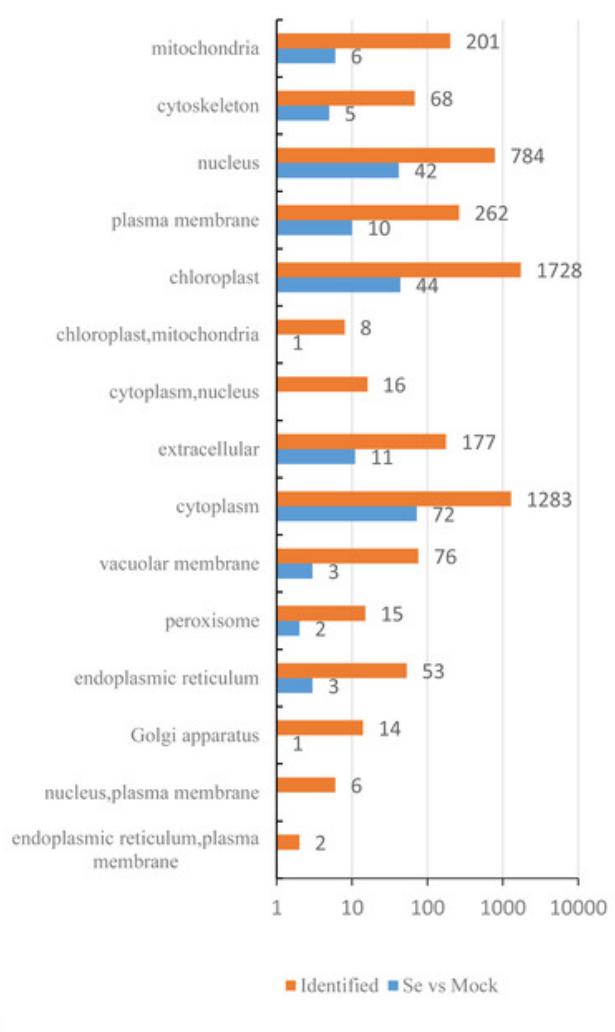




\section{Figure 3}

Impacts Se stress treatment on proteome levels in pepper.

(A) Expression profiles of the DEPs response to Se stress. (B-D) All DEPs were analyzed and clustered into three major Clusters by K-means method. (E) Volcano plot of DEPs. (F).The numbers of up- and down-regulated proteins in the Se treatment seedlings compared to the mock seedlings.

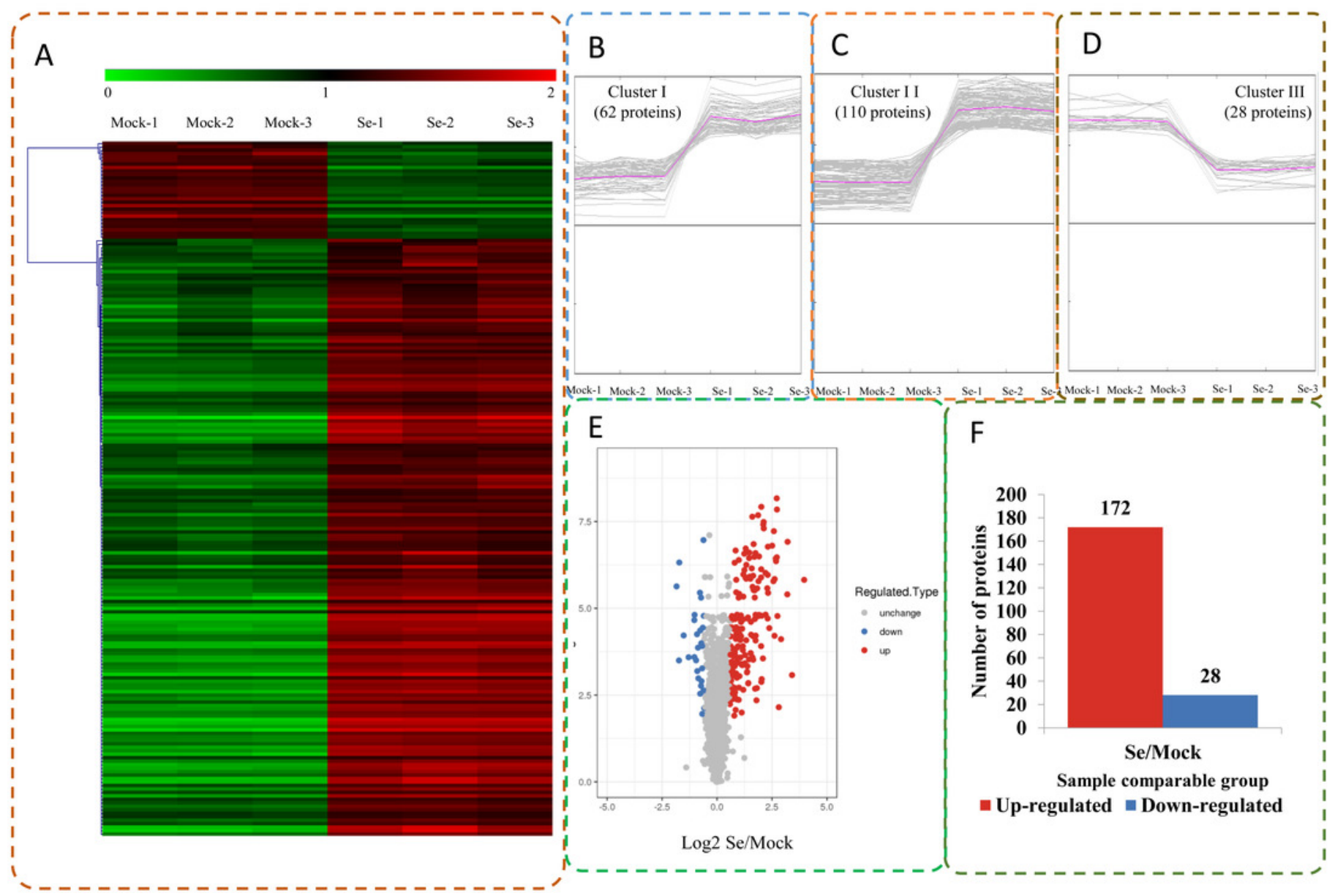




\section{Figure 4}

\section{KOG functional classification chart of Differentially expressed proteins.}

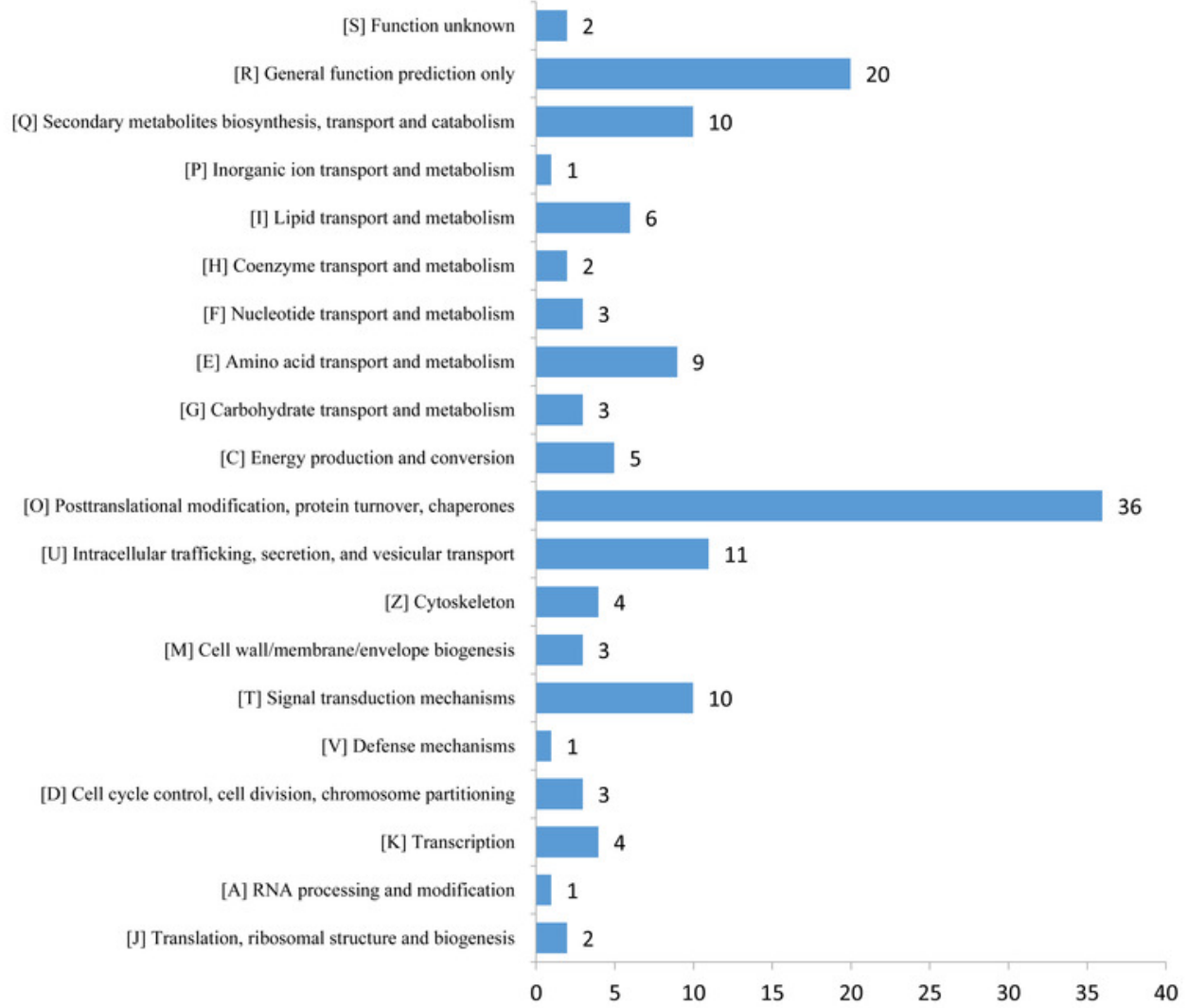


Figure 5

GO enrichment analysis of DEPs.

Distribution of the up-regulated (A) and down-regulated (B) proteins with GO enrichment analysis.

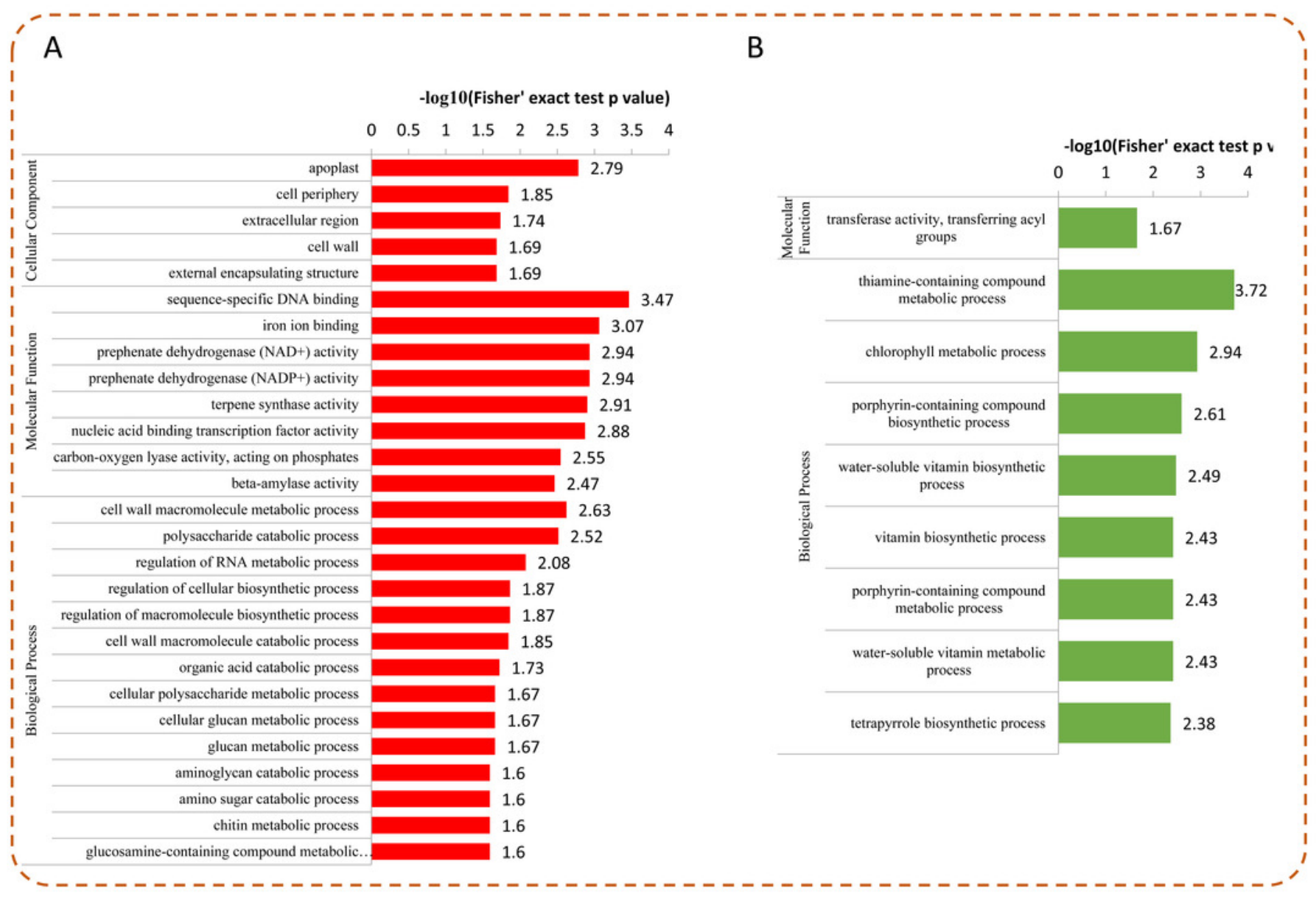


Figure 6

KEGG enrichment analysis of the DEPs in pepper after Se stress treatment.

(A) Significantly enriched KEGG terms of the up-regulated proteins. (B) Significantly enriched KEGG terms of the down-regulated proteins.

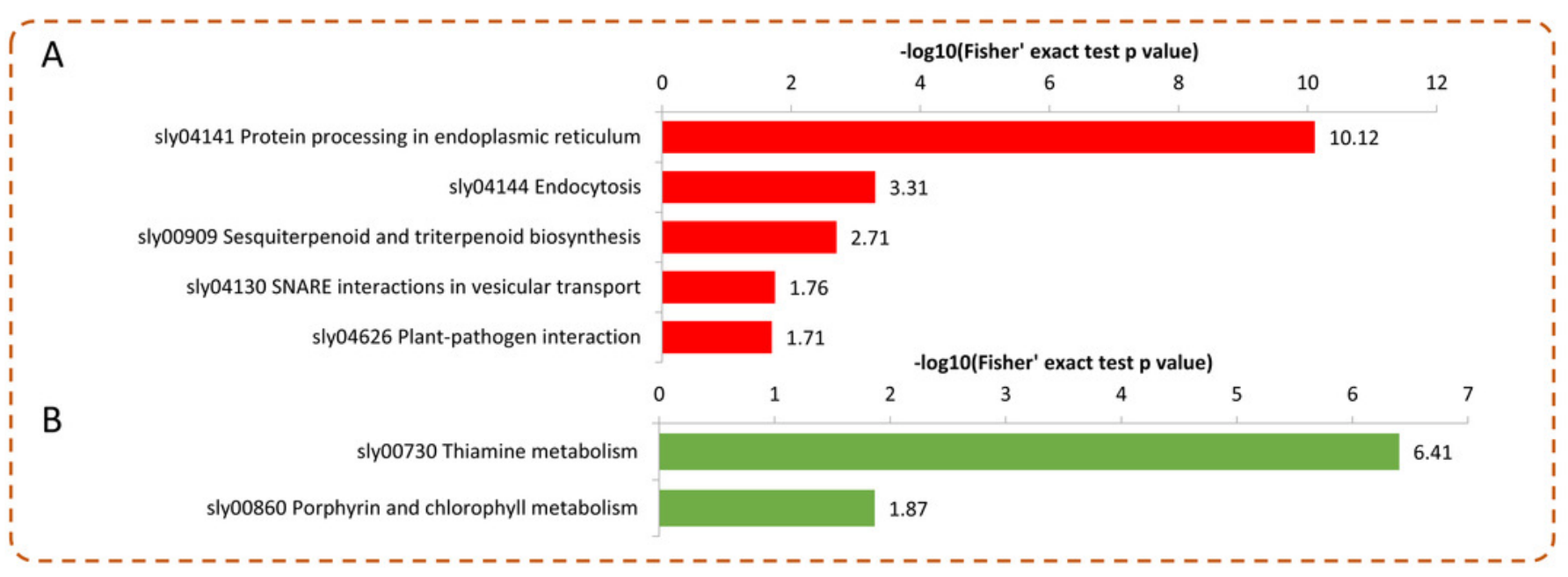


Figure 7

Domain enrichment analysis of the DEPs in pepper after Se stress treatment.

(A) Protein domain enrichment bubble plot of differentially expressed proteins. (B) The accumulation of HSP proteins after Se stress treatment.

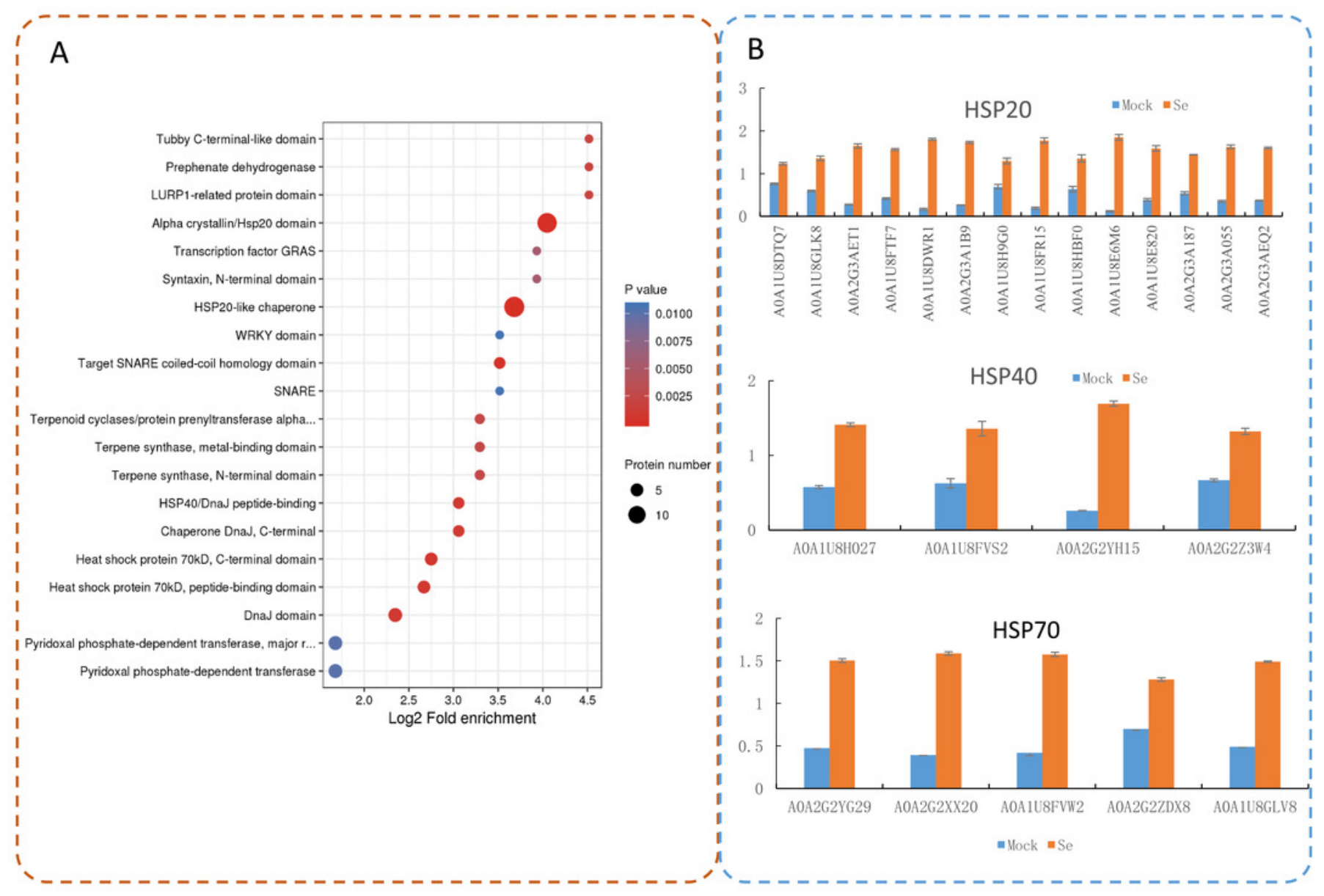




\section{Table 1 (on next page)}

Table 1 Identification of the DEPs involved in metabolic pathways 


\begin{tabular}{lcccc}
\hline $\begin{array}{c}\text { Protein } \\
\text { accession }\end{array}$ & Protein description & Ratio & P value & $\begin{array}{c}\text { MW } \\
{[\mathrm{kDa}]}\end{array}$ \\
\hline $\begin{array}{c}\text { Butanoate metabolism } \\
\text { A0A1U8E166 }\end{array}$ & "Hydroxymethylglutaryl-CoA lyase, mitochondrial & 1.788 & 0.00039971 & 45.332 \\
A0A2G2YVX0 & Glutamate decarboxylase & 4.962 & $1.6822 \mathrm{E}-07$ & 59.504 \\
Cysteine and methionine metabolism & L-lactate dehydrogenase & 4.042 & 0.00132081 & 37.54 \\
A0A2G3AEL6 & 1-aminocyclopropane-1-carboxylate synthase & 3.506 & 0.00199508 & 54.867 \\
A0A2G3ADN2 & 1-aminocyclopropane-1-carboxylate oxidase 4 & 1.601 & $1.67031 \mathrm{E}-05$ & 36.33 \\
A0A1U8FJ05 & 1-aminocyclopropane-1-carboxylate oxidase 1 & 3.69 & $1.6569 \mathrm{E}-06$ & 36.059 \\
A0A1U8FEU9 & Tyrosine aminotransferase & 2.399 & 0.00038372 & 47.177 \\
A0A1U8EYM1 & Arginine decarboxylase & 3.279 & $4.8968 \mathrm{E}-06$ & 78.211 \\
A0A1U8E953 & Proline dehydrogenase & 2.603 & 0.00046451 & 55.32 \\
A0A1U8FBD0 & Lipoxygenase & 2.359 & $2.6782 \mathrm{E}-07$ & 102.6 \\
A0A2G2ZVP6 & Glyoxysomal fatty acid beta-oxidation & 4.247 & 0.00027878 & 101.81 \\
A0A2G2YXE3 & multifunctional protein MFP-a & &
\end{tabular}

Glycerophospholipid metabolism

A0A2G2YY43 Glycerol-3-phosphate 2-O-acyltransferase $4 \quad 0.625 \quad 0.00053958 \quad 51.031$

A0A1U8G1E3 Glycerophosphodiester phosphodiesterase GDPD2 $2.767 \quad 2.4846 \mathrm{E}-07 \quad 42.766$

A0A1U8H0F1 $\quad$ Glycerol-3-phosphate acyltransferase $5 \quad 2.083 \quad 0.00026256 \quad 55.181$

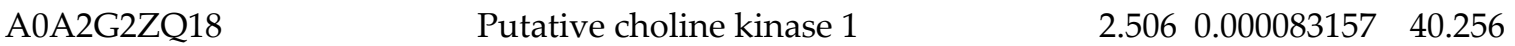

Linoleic acid metabolism

$\begin{array}{lllll}\text { A0A2G2ZVP6 } & \text { Lipoxygenase } & 2.359 & 2.6782 \mathrm{E}-07 & 102.6 \\ \text { A0A2G2ZBY6 } & \text { Lipoxygenase } & 3.646 & 2.0736 \mathrm{E}-08 & 97.904\end{array}$

Phenylpropanoid biosynthesis

$\begin{array}{lcccc}\text { A0A2G2YQ27 } & \text { Phenylalanine ammonia-lyase } & 4.291 & 3.8524 \mathrm{E}-08 & 78.308 \\ \text { A0A1U8DW23 } & \text { Peroxidase } & 1.569 & 0.000022029 & 36.129 \\ \text { A0A2G2YUF1 } & \text { Retinal dehydrogenase 1 } & 2.003 & 3.8333 \mathrm{E}-06 & 54.692 \\ \text { A0A2G3A835 } & \text { Caffeoyl-CoA O-methyltransferase 1 } & 1.769 & 0.000020361 & 27.232\end{array}$

Phenylalanine, tyrosine and tryptophan biosynthesis

$\begin{array}{ccccc}\text { A0A1U8FBU3 } & \text { "Arogenate dehydrogenase 1, chloroplastic } & 1.76 & 0.0026381 & 42.449 \\ \text { A0A1U8EYM1 } & \text { Tyrosine aminotransferase } & 2.399 & 0.00038372 & 47.177 \\ \text { A0A2G2YRI9 } & \text { "Arogenate dehydrogenase 1 } & 2.856 & 1.4056 \mathrm{E}-06 & 45.635\end{array}$

Ubiquinone and other terpenoid-quinone biosynthesis

A0A1U8FWD5 Putative NAD(P)H dehydrogenase (Quinone) FQR1like 1

$1.719 \quad 0.0124045 \quad 21.674$ 
A0A1U8EYM1

Tyrosine aminotransferase

Sesquiterpenoid and triterpenoid biosynthesis

A0A1U8HFR8

Vetispiradiene synthase 1

A0A1U8EWI6

Uncharacterized protein

Thiamine metabolism

A0A2G3ALC4

A0A1U8FC73

A0A2G3A131

A0A2G2Z8I0
"1-deoxy-D-xylulose-5-phosphate synthase, chloroplastic

"Thiamine thiazole synthase, chloroplastic

Adenylate kinase 4

Phosphomethylpyrimidine synthase $\begin{array}{lll}2.399 & 0.00038372 & 47.177\end{array}$

$6.712 \quad 1.67142 \mathrm{E}-05 \quad 64.165$

3.689 1.67363E-05 56.854

$0.607 \quad 0.000102066 \quad 76.896$

$\begin{array}{lll}0.548 & 0.000136529 & 38.071\end{array}$

$0.604 \quad 4.9351 \mathrm{E}-06 \quad 26.487$

$\begin{array}{lll}0.612 & 0.00125775 & 70.093\end{array}$

1 